\title{
Lorentz estimates for asymptotically regular fully nonlinear parabolic equations
}

\author{
Junjie Zhang* Shenzhou Zheng ${ }^{\dagger}$
}

June 21, 2017

\begin{abstract}
We prove a global Lorentz estimate of the Hessian of strong solutions to the Cauchy-Dirichlet problem for a class of fully nonlinear parabolic equations with asymptotically regular nonlinearity over a bounded $C^{1,1}$ domain. Here, we mainly assume that the associated regular nonlinearity satisfies uniformly parabolicity and the $(\delta, R)$-vanishing condition, and the approach of constructing a regular problem by an appropriate transformation is employed.
\end{abstract}

MR(2010) Subject Classification: 35D35; 35K55

Key words: fully nonlinear parabolic equations; asymptotically regular; strong solutions; $(\delta, R)$-vanishing condition; Lorentz spaces

\section{Introduction}

Let $\Omega_{T}=\Omega \times[0, T] \subset \mathbb{R}^{n+1}$ be a parabolic cylinder with a bounded $C^{1,1}$ smooth domain $\Omega \subset \mathbb{R}^{n}$ for $n \geq 1$ and $0<T<\infty$. Throughout the paper, we denote a typical point by $z=(x, t)=\left(x^{1}, x^{2}, \cdots, x^{n}, t\right) \in \mathbb{R}^{n+1}$. The aim of this paper is to prove a global Lorentz estimate of the Hessian of strong solutions to the following zero initial-boundary problem for fully nonlinear parabolic equations:

$$
\begin{cases}u_{t}+F\left(D^{2} u, x, t\right)=f(x, t), & \text { in } \Omega_{T}, \\ u=0, & \text { on } \partial \Omega_{T},\end{cases}
$$

where $\partial \Omega_{T}=(\partial \Omega \times[0, T]) \cup(\Omega \times\{t=0\})$ and the nonlinearity $F\left(D^{2} u, x, t\right): \mathcal{S}(n) \times \Omega \times[0, T] \rightarrow \mathbb{R}$ is asymptotically regular nonlinearity (see Definition 2.2 below), and $\mathcal{S}(n)$ denotes the set of $n \times n$ symmetric matrices.

As we know, the study of second order parabolic equations plays a fundamental role in rather wide fields of partial differential equations, and it is deeply related to the development of several fields in applied and pure mathematics, such as differential geometry, functional and harmonic analysis, mechanics and so on. In this paper, we are mainly interested in the Cauchy-Dirichlet problem of fully nonlinear parabolic equations with asymptotically regular nonlinearity. At this point, Krylov and Safonov's works $[17,18]$ on linear nondivergence elliptic equations set the beginning of the study of the regularity theory for fully nonlinear elliptic and parabolic equations. Since then there arises a large of literature on the Calderón-Zygmund theory

\footnotetext{
*Department of Mathematics, Beijing Jiaotong University, Beijing 100044, China. email:junjiezhang@bjtu.edu.cn.

Corresponding author: 1 Department of Mathematics, Beijing Jiaotong University, Beijing 100044, China. email:shzhzheng@bjtu.edu.cn. ${ }^{2}$ BCAM-Basque Center for Applied Mathematics, Alameda de Mazarredo 14, 48009 Bilbao,Spain.
} 
of nondivergence equations. It is worth noting that Caffarelli [7] first gave an interior $W^{2, p}$ a priori estimate for the viscosity solution of

$$
F\left(D^{2} u, x\right)=f(x), \quad \text { in } B_{1}=B_{1}(0)
$$

under a small oscillation of $F$ in the variable $x$ and the $C^{1,1}$ estimates for the homogeneous equations with constant coefficients $F\left(D^{2} w, x_{0}\right)=0$, by using the Aleksandrov-Bakel'man-Pucci a priori estimate, a covering argument and the Harnack inequality from Krylov-Safonov, also see [8]. Recently, Winter [23] proved a boundary $W^{2, p}$ estimates for the associated elliptic boundary-value problem

$$
\begin{cases}F\left(D^{2} u, x\right)=f(x), & \text { in } B_{1}^{+}, \\ u=0, & \text { on } B_{1} \cap\left\{x_{n}=0\right\}\end{cases}
$$

and demonstrated a global $W^{2, p}$ estimate when the boundary of the domain is additionally smooth enough. As for the parabolic settings, Wang in [22] extended Caffarelli's interior estimate result to that of fully nonlinear parabolic equations by a rather geometrical technique in [7, 23]. Recently, Krylov used in [15] a different but simpler and faster method to investigate Bellman's equations with VMO "coefficients". After that, Dong-Krylov-Li [13] studied a generalized fully nonlinear parabolic equations

$$
u_{t}+F\left(D^{2} u, x, t\right)+G\left(D^{2} u, D u, x, t\right)=0
$$

with VMO "coefficients"

$$
f_{Q_{r}}\left|F\left(D^{2} u, x, t\right)-\bar{F}\left(D^{2} u\right)\right| d x d t \leq \delta, \quad \bar{F}\left(D^{2} u\right)=f_{Q_{r}} F\left(D^{2} u, x, t\right) d x d t
$$

under the assumption that $F\left(D^{2} u, x, t\right)$ is convex with respect to $D^{2} u$, and established a global $W_{p}^{2,1}$ for $p>n+1$ estimate in a bounded $C^{1,1}$ smooth domain. Furthermore, the convexity assumption on $F\left(D^{2} u, x, t\right)$ can be replaced by some relaxed convexity assumptions, for details see Krylov's work [16]. Additionally, we also proved in [25] an interior Lorentz estimate of the Hessian of strong solutions to fully nonlinear parabolic equations $u_{t}+F\left(D^{2} u, x, t\right)=f(x, t)$ by way of the large- $M$-inequality principle introduced by AcerbiMingione [1], under the main assumption that the associated nonlinearities satisfy uniformly parabolic condition and the $(\delta, R)$-vanishing conditions. For more information on this topic of fully nonlinear problems, see $[4,9,10,12]$.

Note that the concept of asymptotically regular was originated in Chipot-Evans's work [11]. Since then there are many works on this asymptotically regular topic regarding elliptic and parabolic problems. For example, Scheven-Schmidt [20] established a local Calderón-Zygmund estimate for asymptotically regular elliptic problems of $p$-Laplacian type for $1<p<\infty$. Scheven et al [21, 14] further proved a local and a global partial Lipschitz regularities of minimizers, respectively. Later, Kuusi and Mingione [19] extended the results in $[21,14]$ to parabolic systems via a new perturbation argument. Very recently, Byun-Oh-Wang [5] studied the global Calderón-Zygmund estimates for nonhomogeneous asymptotically regular elliptic and parabolic problems of divergence form in the Reifenberg flat domain, see also [3]. In [5], they introduced an approach of converting the given asymptotically regular problems to suitable regular ones. Adapting this approach, we in [24] further considered an interior Lorentz estimate for nonlinear parabolic obstacle problems of divergence type with asymptotically regular nonlinearities due to Baroni's work on regular obstacle parabolic problems [2]. Byun et al [6] also proved a global $W^{2, p}$ estimates for viscosity solutions to both asymptotically fully nonlinear elliptic equations in a bounded $C^{1,1}$ domain.

Inspired by [5, 25], we will discuss a global Lorentz estimate of strong solutions to the initial-boundary problem for fully nonlinear parabolic equations over a $C^{1,1}$ bounded domain. In particular, we want to show that the gradient with respect to time variable and the Hessian are as Lorentz integrable as the nonhomogeneous term when the associated nonlinearity is asymptotically regular, which shows that it has a more 
general kind of parabolic behavior near infinity. Our key point in this paper is the construction of a new regular nonlinearity by an appropriate transformation.

The rest of the paper is organized as follows. In section 2, we introduce the definition of asymptotically regular operators and state the main result. In section 3, we first give a proof of global Lorentz estimates for regular fully nonlinear parabolic problem. Then we discuss an appropriate transformation of a given asymptotically regular problem to a regular one, and prove our main conclusion based on the above global result for regular problem.

\section{Basic hypotheses and main result}

We mainly focus on the case that $F(M, x, t)$ is asymptotically regular while considering the initial-boundary problem (1.1). To state our main result, we need recall some basic assumptions. Our consideration is the case that it is getting closer to some real-valued function $G(M, x, t)$ as $\|M\|$ goes to infinity, where $G(M, x, t)$ satisfies the following assumptions:

- H1. $G(M, x, t): \mathcal{S}(n) \times \Omega \times[0, T] \rightarrow \mathbb{R}$ is uniformly parabolic if there exist constants $0<\lambda \leq \Lambda<\infty$ such that for any $M, N \in \mathcal{S}(n)$ and almost all $z=(x, t) \in \Omega_{T}$,

$$
\lambda\|N\| \leq G(M+N, x, t)-G(M, x, t) \leq \Lambda\|N\|, \quad \forall N \geq 0 ;
$$

where we write $N \geq 0$ whenever $N$ is a nonnegative definite symmetric matrix.

- H2. $G(M, x, t)$ is convex and positive homogeneous of degree one in $M$.

- H3. $\left((\delta, R)\right.$-vanishing) Let $\left(x_{0}, t_{0}\right) \in \Omega_{T}$ be fixed and $(x, t) \in \Omega_{T}$. We say $G(M, x, t)$ is $(\delta, R)$-vanishing, if

$$
\sup _{0<r \leq R} \sup _{(y, s) \in \mathbb{R}^{n+1}}\left(f_{Q_{r}(y, s)}\left|\beta_{G}\left(x, t, x_{0}, t_{0}\right)\right|^{n+1} d x d t\right)^{\frac{1}{n+1}} \leq \delta,
$$

where

$$
\beta_{G}\left(x, t, x_{0}, t_{0}\right)=\sup _{M \in \mathcal{S} \backslash\{0\}} \frac{\left|G(M, x, t)-G\left(M, x_{0}, t_{0}\right)\right|}{\|M\|} .
$$

Remark 2.1 (i) $\|N\|$ denotes the $\left(L^{2}, L^{2}\right)$-norm of $N$, i.e. $\|N\|:=\sup _{|x|=1}|N x|$. Therefore, $\|N\|$ is equal to the maximum eigenvalue of $N$ whenever $N \geq 0$.

(ii) The uniformly parabolic assumption $H 1$ implies that $G(M, x, t)$ is monotone increasing and Lipschitz in $M \in \mathcal{S}(n)$ with constant independent of $x$, $t$. As such, for each $(x, t) \in \Omega_{T}$, it is differentiable almost everywhere with respect to $D^{2} u$, and since it is positive homogeneous of degree one in $D^{2} u$, at any point $D^{2} u$ where it is differentiable we have

$$
G\left(D^{2} u, x, t\right)=D_{i j}^{2} u G_{D_{i j}^{2} u}\left(D^{2} u, x, t\right)
$$

With the basic assumptions H1-H3 above in hand, we are now in a position to introduce the concept of the asymptotically regular condition on $F(M, x, t)$.

Definition 2.2 (asymptotically regular) Let $G(M, x, t)$ satisfy the assumptions H1-H3. Then we say that $F(M, x, t)$ is asymptotically regular to $G(M, x, t)$ if there exists a uniformly bounded nonnegative function $\theta:[0, \infty) \rightarrow[0, \infty]$ such that

$$
\varlimsup_{\|M\| \rightarrow \infty} \theta(M) \leq \delta
$$


and

$$
|F(M, x, t)-G(M, x, t)| \leq \theta(\|M\|)(1+\|M\|)
$$

for almost every $(x, t) \in \Omega_{T}$ and all $M \in \mathcal{S}(n)$.

Remark 2.3 (i) By direct computation, we conclude that

$$
\varlimsup_{\|M\| \rightarrow \infty} \sup _{(x, t) \in \Omega_{T}} \frac{|F(M, x, t)-G(M, x, t)|}{\|M\|} \leq 2 \delta,
$$

namely, for any sufficiently small $\delta>0, F(M, x, t)$ is in a regular range as $\|M\|$ is near infinity.

(ii) The asymptotically regular operator may be not satisfying the uniformly parabolicity. For example, consider the following Bellman operator

$$
F(M, x, t)=\inf _{\alpha \in \mathcal{A}}\left(a_{i j}^{\alpha}(x, t) M_{i j}-f^{\alpha}(x, t)\right),
$$

where $\mathcal{A}$ is any set, $f^{\alpha}(x, t)$ is a real function in $\Omega_{T}$ for each $\alpha \in \mathcal{A}$, and $\left(a_{i j}^{\alpha}(x, t)\right)_{i, j=1}^{n}$ has eigenvalues in $[\lambda, \Lambda]$ for each $z=(x, t) \in \Omega_{T}$ and $\alpha \in \mathcal{A}$. It is easy to see that this operator is uniformly parabolic. Now let

$$
\begin{aligned}
F(M, x, t) & =G(M, x, t)+\sin ^{2}\left(|z| M_{11}^{3}\right) \\
& =\inf _{\alpha \in \mathcal{A}}\left(a_{i j}^{\alpha}(x, t) M_{i j}-f^{\alpha}(x, t)+\sin ^{2}\left(|z| M_{11}^{3}\right)\right) .
\end{aligned}
$$

Then $F(M, x, t)$ is asymptotically regular, but it is not uniformly parabolic.

Next, let us recall some basic concepts and facts concerning Lorentz spaces. The Lorentz space $L^{\gamma, q}\left(\Omega_{T}\right)$ with parameters $1 \leq \gamma<\infty$ and $0<q<\infty$ is the set of measurable functions $g$ defined on $\Omega_{T}$ such that

$$
\|g\|_{L^{\gamma, q}\left(\Omega_{T}\right)}:=\left(q \int_{0}^{\infty}\left(\mu^{\gamma}\left|\left\{\xi \in \Omega_{T}:|g(\xi)|>\mu\right\}\right|\right)^{\frac{q}{\gamma}} \frac{d \mu}{\mu}\right)^{\frac{1}{q}}<+\infty
$$

while for $q=\infty$ the Lorentz space $L^{\gamma, \infty}\left(\Omega_{T}\right)$ is set to be the usual Marcinkiewicz space $\mathcal{M}^{\gamma}\left(\Omega_{T}\right)$ with quasinorm

$$
\|g\|_{L^{\gamma, \infty}\left(\Omega_{T}\right)}=\|g\|_{\mathcal{M}^{\gamma}\left(\Omega_{T}\right)}:=\sup _{\mu>0}\left(\mu^{\gamma}\left|\left\{\xi \in \Omega_{T}:|g(\xi)|>\mu\right\}\right|\right)^{\frac{1}{\gamma}}<\infty .
$$

The local variant of Lorentz spaces is defined in the usual way. It is pointed out that by Fubini's theorem we have

$$
\|g\|_{L^{\gamma}\left(\Omega_{T}\right)}=\left(\gamma \int_{0}^{\infty}\left(\mu^{\gamma}\left|\left\{\xi \in \Omega_{T}:|g(\xi)|>\mu\right\}\right|\right) \frac{d \mu}{\mu}\right)^{\frac{1}{\gamma}}=\|g\|_{L^{\gamma, \gamma}\left(\Omega_{T}\right)},
$$

which implies $L^{\gamma}\left(\Omega_{T}\right)=L^{\gamma, \gamma}\left(\Omega_{T}\right)$.

Given a positive integer $k$, we define the parabolic Sobolev space $W_{p}^{k, 1}\left(\Omega_{T}\right)$ as the set of functions $g \in L^{p}\left(\Omega_{T}\right)$ with their derivatives $D_{t}^{s} D_{x}^{l} g \in L^{p}\left(\Omega_{T}\right)$ for $0 \leq 2 s+l \leq k$ with $s \in\{0,1\}$, moreover the norm of $g \in W_{p}^{k, 1}\left(\Omega_{T}\right)$ is given by

$$
\|g\|_{W_{p}^{k, 1}\left(\Omega_{T}\right)}:=\left(\sum_{j=0}^{k} \sum_{2 s+l=j} \int_{\Omega_{T}}\left|D_{t}^{s} D_{x}^{l} g\right|^{p} d x d t\right)^{\frac{1}{p}} .
$$

In particular, if $\Omega_{T}$ is a bounded set we notice that $L^{p}\left(\Omega_{T}\right)$ for $1<p<\infty$ is continuously embedded in $L^{1}\left(\Omega_{T}\right)$, for details see [26]. Further, the Lorentz Sobolev space $W^{2,1} L^{\gamma, q}\left(\Omega_{T}\right)$ is defined by the set of 
functions $u(x, t)$ in $L^{\gamma, q}\left(\Omega_{T}\right)$ whose distributional derivatives $D_{t}^{s} D_{x}^{l} u$ for $0 \leq 2 s+l \leq 2$ with $s \in\{0,1\}$ also belong to $L^{\gamma, q}\left(\Omega_{T}\right)$, and its norm is naturally given by

$$
\|u\|_{W^{2,1} L^{\gamma, q}\left(\Omega_{T}\right)}:=\sum_{j=0}^{2} \sum_{2 s+l=j}\left\|D_{t}^{s} D_{x}^{l} u\right\|_{L^{\gamma, q}\left(\Omega_{T}\right)} .
$$

Finally let us now summarize our main result as follows.

Theorem 2.4 For $\gamma>n+1$ and $0<q \leq \infty$, let $u \in W_{n+1}^{2,1}$ be a strong solution to the Cauchy-Dirichlet problem (1.1) with $f \in L^{\gamma, q}\left(\Omega_{T}\right)$ and $\partial \Omega \in C^{1,1}$. Then there exists a small positive constant $\delta=\delta(n, \lambda, \Lambda, \gamma, q)$ such that if $F\left(D^{2} u, x, t\right)$ is asymptotically regular with $G\left(D^{2} u, x, t\right)$ satisfying the assumptions $H 1-H 3$, we have $u_{t}, D^{2} u \in L^{\gamma, q}\left(\Omega_{T}\right)$ and

$$
\left\|u_{t}\right\|_{L^{\gamma, q}\left(\Omega_{T}\right)}+\left\|D^{2} u\right\|_{L^{\gamma, q}\left(\Omega_{T}\right)} \leq C\left(\|f\|_{L^{\gamma, q}\left(\Omega_{T}\right)}+1\right),
$$

where $C=C\left(n, \lambda, \Lambda, \gamma, q, \Omega_{T}\right)$ (except in the case $t=\infty$, where $C$ depends only on $\left.n, \lambda, \Lambda, \gamma, \Omega_{T}\right)$.

\section{Proof of the main theorem}

In this section, we are devoted to proving our main result based on the following interior Lorentz estimate for fully nonlinear parabolic equations with nonlinearity $G(M, x, t)$ satisfying the regular assumptions $\mathrm{H} 1-\mathrm{H} 3$.

Proposition 3.1 ([25] Theorem 1.2) Let $\gamma>n+1,0<q \leq \infty$, and $u \in W_{n+1}^{2,1}\left(\Omega_{T}\right)$ be a strong solution of fully nonlinear parabolic equations

$$
u_{t}+G\left(D^{2} u, x, t\right)=f(x, t), \quad \text { in } \Omega_{T},
$$

with $f \in L^{\gamma, q}\left(\Omega_{T}\right)$. Then there exists a small positive constant $\delta=\delta(n, \lambda, \Lambda, \gamma, q)$ such that if $G\left(D^{2} u, x, t\right)$ satisfies assumptions H1-H3, we have $u_{t}, D^{2} u \in L_{\text {loc }}^{\gamma, q}\left(\Omega_{T}\right)$. Moreover, there exists a radii $R_{0}=R_{0}(n, \lambda, \Lambda, \gamma, q)$ such that for each cylinder $Q_{2 R}=Q_{2 R}\left(z_{0}\right) \subset \subset \Omega_{T}$ with $0<R \leq R_{0}$ we have

$$
\left\|u_{t}\right\|_{L^{\gamma, q}\left(Q_{R}\right)}+\left\|D^{2} u\right\|_{L^{\gamma, q}\left(Q_{R}\right)} \leq C\left(\left\|u_{t}\right\|_{L^{n+1}\left(Q_{2 R}\right)}+\left\|D^{2} u\right\|_{L^{n+1}\left(Q_{2 R}\right)}+\||f|+1\|_{L^{\gamma, q}\left(Q_{2 R}\right)}\right),
$$

where $C=C(n, \lambda, \Lambda, \gamma, q)$. In the case $q=\infty$, the constants $C, \delta, R_{0}$ above depend only on $n, \lambda, \Lambda, \gamma$.

Next, we give a local boundary estimates in Lorentz space by using the idea of odd/even extensions over the flat boundary. Fix a point $x_{0} \in \partial \Omega$, we suppose

$$
\partial \Omega \text { is flat near } x_{0} \text {, lying in the plane }\left\{x^{1}=0\right\} \text {. }
$$

Then we may assume there exists an open cylinder $Q_{2 R}\left(z_{0}\right)$ with center $z_{0}=\left(x_{0}, t_{0}\right)$ and radius $2 R$ such that

$$
\left\{\begin{array}{l}
Q_{2 R}^{+}:=Q_{2 R}\left(z_{0}\right) \cap\left\{x^{1} \geq 0\right\} \subset \bar{\Omega}_{T}, \\
Q_{2 R}^{-}:=Q_{2 R}\left(z_{0}\right) \cap\left\{x^{1}<0\right\} \subset \mathbb{R}^{n+1}-\bar{\Omega}_{T} .
\end{array}\right.
$$

We also set $\Gamma_{2 R}=Q_{2 R}\left(z_{0}\right) \cap\left\{x^{1}=0\right\}$. 
Proposition 3.2 For $\gamma>n+1$ and $0<q \leq \infty$, let $u \in W_{n+1}^{2,1}\left(\Omega_{T}\right)$ be any strong solution of local initialboundary value problem

$$
\begin{cases}u_{t}+G\left(D^{2} u, x, t\right)=f(x, t), & \text { in } Q_{2 R}^{+}, \\ u=0, & \text { on } \Gamma_{2 R} \cup\left(B_{2 R}^{+} \times\left\{t_{0}-4 R^{2}\right\}\right)\end{cases}
$$

with $f \in L^{\gamma, q}\left(\Omega_{T}\right)$. Then there exist small positive constants $\delta$ and $R_{0}$ depending only on $n, \lambda, \Lambda, \gamma, q$ such that if $G\left(D^{2} u, x, t\right)$ satisfies assumptions $H 1-H 3$, we have

$$
\left\|u_{t}\right\|_{L^{\gamma, q}\left(Q_{R}^{+}\right)}+\left\|D^{2} u\right\|_{L^{\gamma, q}\left(Q_{R}^{+}\right)} \leq C\left(\left\|u_{t}\right\|_{L^{n+1}\left(Q_{2 R}^{+}\right)}+\left\|D^{2} u\right\|_{L^{n+1}\left(Q_{2 R}^{+}\right)}+\||f|+1\|_{L^{\gamma, q}\left(Q_{2 R}^{+}\right)}\right),
$$

for each half cylinder $Q_{2 R}^{+}$with $0<R \leq R_{0}$, where $C=C(n, \lambda, \Lambda, \gamma, q)$. In the case $q=\infty$, the constants $C, \delta, R_{0}$ above depend only on $n, \lambda, \Lambda, \gamma$.

Proof. As pointed in Remark 2.3 (ii), the assumptions $\mathrm{H} 1$ and $\mathrm{H} 2$ imply that

$$
G\left(D^{2} u, x, t\right)=G_{D_{i j}^{2} u}\left(D^{2} u, x, t\right) D_{i j}^{2} u:=a_{i j}(x, t) D_{i j}^{2} u .
$$

We now define $\hat{u}$ in $Q_{2 R}\left(z_{0}\right)$ with $x_{0}$ on flat boundary by

$$
\hat{u}\left(x^{1}, x^{\prime}, t\right)= \begin{cases}u\left(x^{1}, x^{\prime}, t\right) & \text { if } x^{1} \geq 0 \\ u\left(-x^{1}, x^{\prime}, t\right) & \text { if } x^{1}<0\end{cases}
$$

and extend $a_{i j}(x, t)=a_{i j}\left(x^{1}, x^{\prime}, t\right)$ from $\left\{x^{1} \geq 0\right\}$ to $\left\{x^{1}<0\right\}$ by even or odd reflection, depending on the indices $i$ and $j$. Specifically, when $x^{1} \geq 0, \hat{a}_{i j}(x, t)=a_{i j}(x, t)$; when $x^{1}<0$,

$$
\hat{a}(x, t)= \begin{cases}a_{i j}\left(-x^{1}, x^{\prime}, t\right), & \text { if } i=j=1 \text { or } i, j \in\{2, \cdots, n\}, \\ -a_{i j}\left(-x^{1}, x^{\prime}, t\right), & \text { if } i \in\{2, \cdots, n\} \text { and } j=1 .\end{cases}
$$

Also set $\hat{a}_{1 j}=\hat{a}_{j 1}$. We see that the nonlinearity $\widehat{G}\left(D^{2} \hat{u}, x, t\right)$ satisfy the assumptions H1-H3. Let $\hat{f}$ be the odd extension of $f$ with respect to $x^{1}$, then it is easy to see that $\hat{f} \in L^{\gamma, q}\left(Q_{2 R}\left(z_{0}\right)\right)$. Lemma 3.1 implies that the extended $\hat{u}$ is a strong solution of $\hat{u}_{t}+\widehat{G}\left(D^{2} \hat{u}, x, t\right)=\hat{f}$ in $Q_{2 R}\left(z_{0}\right)$ with the local Lorentz estimates (3.2). Therefore, we can obtain the desired estimates (3.4) by restricting $\hat{u}$ from $Q_{2 R}\left(z_{0}\right)$ to $Q_{2 R}^{+}$.

Based on the above interior and flat boundary Lorentz estimates, we can derive a global result via the standard flattening and covering arguments as usual way.

Theorem 3.3 For $\gamma>n+1$ and $0<q \leq \infty$, let $u \in W_{n+1}^{2,1}$ be a strong solution to Cauchy-Dirichlet problem

$$
\begin{cases}u_{t}+G\left(D^{2} u, x, t\right)=f(x, t), & \text { in } \Omega_{T}, \\ u=0, & \text { on } \partial \Omega_{T},\end{cases}
$$

satisfying assumptions $H 1$-H3 for some small positive constant $\delta=\delta(n, \lambda, \Lambda, \gamma, q)$ and $f \in L^{\gamma, q}\left(\Omega_{T}\right), \partial \Omega \in$ $C^{1,1}$. Then there exist positive constant $C=C\left(n, \lambda, \Lambda, \gamma, q, \Omega_{T}\right)$ such that $u_{t}, D^{2} u \in L^{\gamma, q}\left(\Omega_{T}\right)$ and

$$
\left\|u_{t}\right\|_{L^{\gamma, q}\left(\Omega_{T}\right)}+\left\|D^{2} u\right\|_{L^{\gamma, q}\left(\Omega_{T}\right)} \leq C\|f\|_{L^{\gamma, q}\left(\Omega_{T}\right)} .
$$

While $q=\infty$, the constants $C, \delta$ depend only on $n, \lambda, \Lambda, \gamma, \Omega_{T}$. 
Proof. (1). First we claim that

$$
u \in W^{2,1} L^{\gamma, q}\left(\Omega_{T}\right),
$$

which will be strictly proved in the step 3 . For fixed any point $x_{0} \in \partial \Omega$, we now flatten the boundary near $x_{0}$ in order to apply the flat boundary estimates (3.4). Thanks to the assumption $\partial \Omega \in C^{1,1}$, there exists a neighborhood $\mathcal{N}_{0} \ni x_{0}$ and a $C^{1,1}$-diffeomorphism $\Phi: \mathcal{N}_{0} \rightarrow B_{2 R}(0)$ such that

$$
\Phi\left(x_{0}\right)=0, \quad \text { and } \quad \Phi\left(\mathcal{N}_{0} \cap \Omega\right)=B_{2 R}^{+} .
$$

We write $y=\Phi(x), x \in \mathcal{N}_{0} \cap \Omega$, and define $\Psi=\Phi^{-1}$, then $x=\Psi(y)$. Define $\tilde{u}(y, t)=u(\Psi(y), t)=u(x, t)$ for $y \in B_{2 R}^{+}$. Then it is readily checked that $\tilde{u} \in W_{n+1}^{2,1}\left(Q_{2 R}^{+}\right)$is a strong solution of the following flat initialboundary problems

$$
\begin{cases}\tilde{u}_{t}+\widetilde{F}\left(D^{2} \tilde{u}, y, t\right)=\tilde{f}(y, t), & \text { in } Q_{2 R}^{+}, \\ \tilde{u}=0, & \text { on } \Gamma_{2 R} \cup\left(B_{2 R}^{+} \times\left\{-R^{2}\right\}\right),\end{cases}
$$

where

$$
\widetilde{F}\left(D^{2} \tilde{u}, y, t\right):=F\left(\left(D \Phi^{T} \circ \Psi\right) D^{2} \tilde{u}(D \Phi \circ \Psi)+D \tilde{u}\left(D^{2} \Phi \circ \Psi\right), \Psi(y), t\right),
$$

and

$$
\tilde{f}(y, t):=f(\Psi(y), t) .
$$

Note that $\widetilde{F}$ is convex in $D^{2} \tilde{u}$ and $\widetilde{F}(0, y, t)=0$, we readily see that $\beta_{\widetilde{F}}\left(y, t, y_{0}, t_{0}\right) \leq C(\Phi) \beta_{F}\left(\Psi(y), t, \Psi\left(y_{0}\right), t_{0}\right)$ for any $(y, t),\left(y_{0}, t_{0}\right) \in Q_{2 R}^{+}$; and $\widetilde{F}$ satisfies the assumptions H1-H3 with different positive constants. Therefore, all hypotheses of Lemma 3.2 are satisfied, and it yields

$$
\left\|\tilde{u}_{t}\right\|_{L^{\gamma, q}\left(Q_{R}^{+}\right)}+\left\|D^{2} \tilde{u}\right\|_{L^{\gamma, q}\left(Q_{R}^{+}\right)} \leq C\left(\left\|\tilde{u}_{t}\right\|_{L^{n+1}\left(Q_{2 R}^{+}\right)}+\left\|D^{2} \tilde{u}\right\|_{L^{n+1}\left(Q_{2 R}^{+}\right)}+\|\tilde{f}\|_{L^{\gamma, q}\left(Q_{2 R}^{+}\right)}\right) .
$$

Converting back to the original $x$-variables, we conclude

$$
\left\|u_{t}\right\|_{L^{\gamma, q}\left(V_{R}\right)}+\left\|D^{2} u\right\|_{L^{\gamma, q}\left(V_{R}\right)} \leq C\left(\left\|u_{t}\right\|_{L^{n+1}\left(V_{2 R}\right)}+\left\|D^{2} u\right\|_{L^{n+1}\left(V_{2 R}\right)}+\|f\|_{L^{\gamma, q}\left(V_{2 R}\right)}\right),
$$

where $V_{R}=\Psi\left(B_{R}^{+}\right) \times\left(t_{0}-R^{2}, t_{0}\right)=\left(\mathcal{N}_{0} \cap \Omega\right) \times\left(t_{0}-R^{2}, t_{0}\right)$. Since $\partial \Omega$ is compact, there exist finitely many points $x_{1}, x_{2}, \cdots, x_{N} \in \partial \Omega$, and open neighborhoods $\mathcal{N}_{i}$ defined as $\mathcal{N}_{0}$ above such that $\partial \Omega \subset \cup_{i=1}^{N} \mathcal{N}_{i}$. Take $\mathcal{N}_{N+1} \subset \subset \Omega$ so that $\Omega=\cup_{i=1}^{N+1} \mathcal{N}_{i}$, and let $\left\{\zeta_{i}\right\}_{i=1}^{N+1}$ be a partition of unity associated with $\Omega=\cup_{i=1}^{N+1} \mathcal{N}_{i}$. Write $u=\sum_{i=1}^{N+1} \zeta_{i} u_{i}$, then utilizing the boundary Lorentz estimates (3.8) (with $u_{i}$ in place of $u$ ) and the interior Lorentz estimate (3.2), we obtain

$$
\left\|u_{t}\right\|_{L^{\gamma, q}\left(\Omega_{T}\right)}+\left\|D^{2} u\right\|_{L^{\gamma, q}\left(\Omega_{T}\right)} \leq C\left(\left\|u_{t}\right\|_{L^{n+1}\left(\Omega_{T}\right)}+\left\|D^{2} u\right\|_{L^{n+1}\left(\Omega_{T}\right)}+\|f\|_{L^{\gamma, q}\left(\Omega_{T}\right)}\right),
$$

for some positive constant $C$ depending on $n, \lambda, \Lambda, \gamma, q, \Omega_{T}$, which implies

$$
\|u\|_{W^{2,1} L^{\gamma, q}\left(\Omega_{T}\right)} \leq C\left(\|D u\|_{L^{\gamma, q}\left(\Omega_{T}\right)}+\left\|u_{t}\right\|_{L^{n+1}\left(\Omega_{T}\right)}+\left\|D^{2} u\right\|_{L^{n+1}\left(\Omega_{T}\right)}+\|f\|_{L^{\gamma, q}\left(\Omega_{T}\right)}\right) .
$$

(2). We next show that there exists a positive constant $C$ such that

$$
\|u\|_{W^{2,1} L^{\gamma, q}\left(\Omega_{T}\right)} \leq C\|f\|_{L^{\gamma, q}\left(\Omega_{T}\right)} .
$$

We do it by contradiction. Indeed, if (3.11) is not true, then there exists a sequence $\left\{u_{k}\right\}_{k=1}^{\infty}$ and $\left\{f_{k}\right\}_{k=1}^{\infty}$ such that $u_{k}$ is a strong solution of the following problem:

$$
\begin{cases}\left(u_{k}\right)_{t}+F\left(D^{2} u_{k}, x, t\right)=f_{k}(x, t), & \text { in } \Omega_{T}, \\ u_{k}=0, & \text { on } \partial \Omega_{T},\end{cases}
$$


satisfying

$$
\left\|u_{k}\right\|_{W^{2,1} L^{\gamma, q}\left(\Omega_{T}\right)}>k\left\|f_{k}\right\|_{L^{\gamma, q}\left(\Omega_{T}\right)}, \quad \text { for any } k \geq 1 .
$$

Without loss of generality, we may suppose that

$$
\left\|u_{k}\right\|_{W^{2,1} L^{\gamma, q}\left(\Omega_{T}\right)}=1 .
$$

Then it follows from (3.12) that

$$
\left\|f_{k}\right\|_{L^{\gamma, q}\left(\Omega_{T}\right)}<\frac{1}{k} \rightarrow 0, \quad \text { as } k \rightarrow \infty .
$$

Since $\left\{u_{k}\right\}_{k=1}^{\infty}$ is uniformly bounded in $W^{2,1} L^{\gamma, q}\left(\Omega_{T}\right)$, there exists a subsequence, which be still denoted by $\left\{u_{k}\right\}_{k=1}^{\infty}$, and a function $u_{0} \in W^{2,1} L^{\gamma, q}\left(\Omega_{T}\right)$, such that

$$
u_{k} \rightarrow u_{0} \quad \text { weakly in } W^{2,1} L^{\gamma, q}\left(\Omega_{T}\right), \quad u_{k} \rightarrow u_{0} \quad \text { in } L^{\gamma, q}\left(\Omega_{T}\right), \quad \text { as } k \rightarrow \infty .
$$

It is easy to check that $u_{0}$ is a strong solution of

$$
\begin{cases}\left(u_{0}\right)_{t}+F\left(D^{2} u_{0}, x, t\right)=0, & \text { in } \Omega_{T}, \\ u_{0}=0, & \text { on } \partial \Omega_{T} .\end{cases}
$$

Accordingly, $u_{0}=0$ due to the uniqueness of strong solutions to zero initial-boundary problem (3.16), so it follows from (3.14) and (3.15) that

$$
\begin{array}{ll}
f_{k} \rightarrow 0 & \text { in } L^{\gamma, q}\left(\Omega_{T}\right), \\
u_{k} \rightarrow 0 & \text { weakly in } W^{2,1} L^{\gamma, q}\left(\Omega_{T}\right), \\
u_{k} \rightarrow 0 & \text { in } L^{\gamma, q}\left(\Omega_{T}\right),
\end{array}
$$

as $k \rightarrow \infty$. Note that $W^{2,1} L^{\gamma, q}\left(\Omega_{T}\right) \hookrightarrow W^{2,1} L^{n+1}\left(\Omega_{T}\right)$ due to the fact that $\gamma>n+1$, hence

$$
\left\|u_{k}\right\|_{L^{n+1}\left(\Omega_{T}\right)} \rightarrow 0, \quad\left\|D u_{k}\right\|_{L^{n+1}\left(\Omega_{T}\right)} \rightarrow 0, \quad \text { as } k \rightarrow \infty .
$$

Moreover, letting the measure $v:=d x d t$, we see that

$$
D u_{k} \rightarrow 0 \quad v-\text { a.e. in } \Omega_{T} \quad \text { as } k \rightarrow \infty \quad \text { (up to subsequence), }
$$

which implies

$$
\left|\left\{(x, t) \in \Omega_{T}:\left|D u_{k}\right|>\mu\right\}\right| \rightarrow 0 \quad \text { for any } \mu>0 \quad \text { as } k \rightarrow \infty .
$$

Since $\left\|D u_{k}\right\|_{L^{\gamma, q}\left(\Omega_{T}\right)}$ is uniformly bounded due to (3.13), by Lebesgue Dominated Convergence Theorem we get

$$
D u_{k} \rightarrow 0 \quad \text { in } L^{\gamma, q}\left(\Omega_{T}\right) \quad \text { as } k \rightarrow \infty .
$$

Combining (3.10) (3.13) (3.17) (3.18) and (3.19), it yields

$$
1 \leq C\left(\left\|D u_{k}\right\|_{L^{\gamma, q}\left(\Omega_{T}\right)}+\left\|\left(u_{k}\right)_{t}\right\|_{L^{n+1}\left(\Omega_{T}\right)}+\left\|D^{2} u_{k}\right\|_{L^{n+1}\left(\Omega_{T}\right)}+\left\|f_{k}\right\|_{L^{\gamma, q}\left(\Omega_{T}\right)}\right) \rightarrow 0 \quad \text { as } k \rightarrow \infty,
$$

which is a contradiction. So (3.11) is proved.

(3). Finally, we focus on proving the claim (3.7) by using the uniqueness property of strong solutions to problem (3.5). Choosing a sequence $\left\{f_{k}\right\}_{k=1}^{\infty}$ in $C_{0}^{\infty}(\Omega)$ satisfying

$$
f_{k} \rightarrow f \quad \text { in } \quad L^{\gamma, q}\left(\Omega_{T}\right) \quad \text { as } k \rightarrow \infty \quad \text { and } \quad\left\|f_{k}\right\|_{L^{\gamma, q}\left(\Omega_{T}\right)} \leq C\|f\|_{L^{\gamma, q}\left(\Omega_{T}\right)}
$$


we observe that for each $k \in \mathbb{N}$ there exists a unique strong solution $u_{k}$ of

$$
\begin{cases}\left(u_{k}\right)_{t}+F\left(D^{2} u_{k}, x, t\right)=f_{k}(x, t), & \text { in } \Omega_{T}, \\ u_{k}=0, & \text { on } \partial \Omega_{T} .\end{cases}
$$

Obviously, these strong solutions $u_{k}$ belong to $W^{2,1} L^{\gamma, q}\left(\Omega_{T}\right)$. Since (3.11) and (3.20), there exists a positive constant $C$, independent of $k$, such that

$$
\left\|u_{k}\right\|_{W^{2,1} L^{\gamma, q}\left(\Omega_{T}\right)} \leq C\left\|f_{k}\right\|_{L^{\gamma, q}\left(\Omega_{T}\right)} \leq C\|f\|_{L^{\gamma, q}\left(\Omega_{T}\right)} .
$$

This shows that $\left\{u_{k}\right\}_{k=1}^{\infty}$ is uniformly bounded in $W^{2,1} L^{\gamma, q}\left(\Omega_{T}\right)$, and so there exists a subsequence, which is still denoted by $\left\{u_{k}\right\}_{k=1}^{\infty}$, and a function $v \in W^{2,1} L^{\gamma, q}\left(\Omega_{T}\right)$ such that

$$
u_{k} \rightarrow v \quad \text { weakly in } W^{2,1} L^{\gamma, q}\left(\Omega_{T}\right) \quad \text { as } k \rightarrow \infty .
$$

Therefore,

$$
\|v\|_{W^{2,1} L^{\gamma, q}\left(\Omega_{T}\right)} \leq \liminf _{k \rightarrow \infty}\left\|u_{k}\right\|_{W^{2,1} L^{\gamma, q}\left(\Omega_{T}\right)} \leq C\|f\|_{L^{\gamma, q}\left(\Omega_{T}\right)} .
$$

We can also check that $v$ is a strong solution to the initial-boundary problem (3.5), then $u=v$ due to the uniqueness of strong solutions of problem (3.5), which implies (3.7). This completes the proof of Theorem 3.3 .

In what follows, the key technique to prove Theorem 2.4 is to use an appropriate transformation to construct a regular problem whose nonlinearity satisfies the assumptions H1-H3. More precisely, we define a real-valued function $H(M, x, t): \mathcal{S}(n) \times \Omega \times[0, T] \rightarrow \mathbb{R}$ by

$$
\|M\| H(M, x, t)=F(M, x, t)-G(M, x, t) .
$$

Then (2.4) implies that for any sufficiently small $\delta>0$, there exists a positive constant $K=K(\delta)$ such that

$$
\|M\| \geq K \Rightarrow|H(M, x, t)| \leq 2 \delta, \quad \forall(x, t) \in \Omega_{T} .
$$

For any fixed point $(x, t) \in \Omega_{T}$, we define a new real-valued function $\widetilde{H}(M, x, t)$ by

$$
\widetilde{H}(M, x, t)= \begin{cases}H(M, x, t), & \text { if }\|M\| \geq K, \\ \frac{\|M\|}{K} H\left(\frac{K}{\|M\|} M, x, t\right), & \text { if }\|M\|<K, \\ 0, & \text { if }\|M\|=0 .\end{cases}
$$

Then $\widetilde{H}(M, x, t)$ is also convex in $M$ and positive homogeneous of degree one in $M$, and that for any $M \in \mathcal{S}(n)$ there holds

$$
|\widetilde{H}(M, x, t)| \leq 2 \delta
$$

uniformly in $(x, t) \in \Omega_{T}$.

Now, we combine (3.23) with (3.25) to derive that for any $M \neq 0$,

$$
\begin{aligned}
F(M, x, t) & =G(M, x, t)+\|M\| H(M, x, t) \\
& =G(M, x, t)+\|M\| \widetilde{H}(M, x, t)+\|M\|(H(M, x, t)-\widetilde{H}(M, x, t)) \\
& =G(M, x, t)+\|M\| \widetilde{H}(M, x, t)+\|M\| \chi_{\{M \in \mathcal{S}(n):\|M\|<K\}}(H(M, x, t)-\widetilde{H}(M, x, t)),
\end{aligned}
$$

where $\chi_{\{M \in \mathcal{S}(n):\|M\|<K\}}$ is the characteristic function on the set $\{M \in \mathcal{S}(n):\|M\|<K\}$. On the other hand, we define $\left.\|M\| H(M, x, t)\right|_{M=0}:=F(0, x, t)-G(0, x, t)$, then the equation (3.27) holds for all $M \in \mathcal{S}(n)$. 
Here, we introduce a new nonlinearity $\widetilde{F}(M, x, t)$, which is regular and transferred from an asymptotically regular one. For a given strong solution $u$ of the initial-boundary problem (1.1), we define

$$
\widetilde{F}(M, x, t): \mathcal{S}(n) \times \Omega \times[0, T] \rightarrow \mathbb{R}
$$

by

$$
\widetilde{F}(M, x, t):=G(M, x, t)+\|M\| \widetilde{H}\left(D^{2} u, x, t\right) .
$$

Then by (3.27) and (3.28), we have

$$
\begin{aligned}
F\left(D^{2} u, x, t\right) & =G\left(D^{2} u, x, t\right)+\left\|D^{2} u\right\| \widetilde{H}\left(D^{2} u, x, t\right)+\left\|D^{2} u\right\| \chi_{\left\{\left\|D^{2} u\right\|<K\right\}}\left(H\left(D^{2} u, x, t\right)-\widetilde{H}\left(D^{2} u, x, t\right)\right) \\
& =\widetilde{F}\left(D^{2} u, x, t\right)+\left\|D^{2} u\right\| \chi_{\left\{\left\|D^{2} u\right\|<K\right\}}\left(H\left(D^{2} u, x, t\right)-\widetilde{H}\left(D^{2} u, x, t\right)\right),
\end{aligned}
$$

where $\chi_{\left\{\left\|D^{2} u\right\|<K\right\}}=\chi_{\left\{(x, t) \in \Omega_{T}:\left\|D^{2} u(x, t)\right\|<K\right\}}$ denotes the characteristic function of the set $\left\{(x, t) \in \Omega_{T}:\left\|D^{2} u(x, t)\right\|<\right.$ $K\}$. Thus (1.1) implies that $u$ is a strong solution of

$$
u_{t}+\widetilde{F}\left(D^{2} u, x, t\right)=f-\left\|D^{2} u\right\| \chi_{\left\{\left\|D^{2} u\right\|<K\right\}}\left(H\left(D^{2} u, x, t\right)-\widetilde{H}\left(D^{2} u, x, t\right)\right):=g \quad \text { in } \Omega_{T} .
$$

We are now in a position to prove the following lemma, which play an important role to verify the main assumptions for the operator $\widetilde{F}(M, x, t)$.

Lemma 3.4 Let $u$ be a strong solution of problem (1.1). Assume that $F(M, x, t)$ is asymptotically regular with $G(M, x, t)$ satisfying the assumptions H1-H3. Then we have (i) If $0<\delta<\frac{\lambda}{4}$, then $\widetilde{F}(M, x, t)$ satisfies the uniformly parabolicity

$$
\tilde{\lambda}\|N\| \leq \widetilde{F}(M+N, x, t)-\widetilde{F}(M, x, t) \leq \widetilde{\Lambda}\|N\|, \quad \forall N \geq 0 .
$$

for almost every $(x, t) \in \Omega_{T}$ and all $M, N \in \mathcal{S}(n)$, where $\tilde{\lambda}=\frac{\lambda}{2}$ and $\widetilde{\Lambda}=\Lambda+\frac{\lambda}{2}$.

(ii) $\widetilde{F}(M, x, t)$ satisfies the $(5 \delta, R)$-vanishing condition.

Proof. (i) Let $0<\delta<\frac{\lambda}{4}$. For any $M, N \in \mathcal{S}(n)$ with $N \geq 0$, it follows from (3.28) that

$$
\widetilde{F}(M+N, x, t)-\widetilde{F}(M, x, t)=G(M+N, x, t)-G(M, x, t)+(\|M+N\|-\|M\|) \widetilde{H}\left(D^{2} u, x, t\right) .
$$

Observe from (3.26) that

$$
\left|\widetilde{H}\left(D^{2} u, x, t\right)\right| \leq 2 \delta,
$$

uniformly with respect to $(x, t) \in \Omega_{T}$. We note that the following triangle inequality

$$
\|M+N\|-\|M\| \leq\|N\| .
$$

Hence, by assumption $\mathrm{H} 1$ we find that

$$
\widetilde{F}(M+N, x, t)-\widetilde{F}(M, x, t) \geq \lambda\|N\|-2 \delta\|N\|=(\lambda-2 \delta)\|N\| \geq \frac{\lambda}{2}\|N\|=\tilde{\lambda}\|N\|,
$$

and

$$
\widetilde{F}(M+N, x, t)-\widetilde{F}(M, x, t) \leq \Lambda\|N\|+2 \delta\|N\|=(\Lambda+2 \delta)\|N\| \leq\left(\Lambda+\frac{\lambda}{2}\right)\|N\|=\widetilde{\Lambda}\|N\|,
$$

where $\tilde{\lambda}=\frac{\lambda}{2}$ and $\widetilde{\Lambda}=\Lambda+\frac{\lambda}{2}$. So (i) is proved.

(ii) Let $0<r \leq R$ and $\left(x_{0}, t_{0}\right) \in \Omega_{T}$. Then for any $(x, t) \in Q_{r}\left(x_{0}, t_{0}\right) \cap \Omega_{T}, M \in \mathcal{S}(n) \backslash\{0\}$ and any $\delta>0$, it follows from (3.28) and (3.26) that

$$
\left|\widetilde{F}(M, x, t)-\widetilde{F}\left(M, x_{0}, t_{0}\right)\right| \leq\left|G(M, x, t)-G\left(M, x_{0}, t_{0}\right)\right|+2 \delta\|M\|+2 \delta\|M\|
$$




$$
=\left|G(M, x, t)-G\left(M, x_{0}, t_{0}\right)\right|+4 \delta\|M\| .
$$

So we derive that

$$
\begin{aligned}
\beta_{\widetilde{F}}\left(x, t, x_{0}, t_{0}\right) & :=\sup _{M \in \mathcal{S}(n) \backslash\{0\}} \frac{\left|\widetilde{F}(M, x, t)-\widetilde{F}\left(M, x_{0}, t_{0}\right)\right|}{\|M\|} \\
& \leq \sup _{M \in \mathcal{S}(n) \backslash\{0\}} \frac{\left|G(M, x, t)-G\left(M, x_{0}, t_{0}\right)\right|}{\|M\|}+4 \delta \\
& =\beta_{G}\left(x, t, x_{0}, t_{0}\right)+4 \delta .
\end{aligned}
$$

Therefore, by (3.32), assumption H3 and the Minkowski inequality, we have

$$
\begin{aligned}
& \left(f_{Q_{r}\left(x_{0}, t_{0}\right) \cap \Omega_{T}} \beta_{\widetilde{F}}\left(x, t, x_{0}, t_{0}\right)^{n+1} d x d t\right)^{\frac{1}{n+1}} \\
\leq & \left(f_{Q_{r}\left(x_{0}, t_{0}\right) \cap \Omega_{T}} \beta_{G}\left(x, t, x_{0}, t_{0}\right)^{n+1} d x d t\right)^{\frac{1}{n+1}}+4 \delta \\
\leq & \delta+4 \delta \\
\leq & 5 \delta,
\end{aligned}
$$

namely, $\widetilde{F}(M, x, t)$ satisfies the $(5 \delta, R)$-vanishing condition. So (ii) is proved.

With global Lorentz estimates regarding the initial-boundary problem of regular Eq.(3.1) in hand, we are now devoted to focusing on our main proof.

The proof of Theorem 2.4. From (3.29) we know that for any given small constant $0<\delta<\min \left\{\frac{\lambda}{4}, 1\right\}$, there exists a positive constant $K>1$ and a real-valued function $\widetilde{H}\left(D^{2} u, x, t\right)$ such that

$$
\left|\widetilde{H}\left(D^{2} u, x, t\right)\right| \leq 2 \delta<2 \text { for all }(x, t) \in \mathbb{R}^{n+1},
$$

and

$$
\begin{cases}u_{t}+\widetilde{F}\left(D^{2} u, x, t\right)=g(x, t), & \text { in } \Omega_{T}, \\ u=0, & \text { on } \partial \Omega_{T}\end{cases}
$$

where

$$
g=f-\left\|D^{2} u\right\| \chi_{\left\{\left\|D^{2} u\right\|<K\right\}}\left(H\left(D^{2} u, x, t\right)-\widetilde{H}\left(D^{2} u, x, t\right)\right) .
$$

By Definition 2.2 we have

$$
\begin{aligned}
\left|\left\|D^{2} u\right\| \chi_{\left.\left\{\left\|D^{2} u\right\|<K\right\}\right\}} H\left(D^{2} u, x, t\right)\right| & =\left|F\left(D^{2} u, x, t\right)-G\left(D^{2} u, x, t\right)\right| \chi_{\left\{\left\|D^{2} u\right\|<K\right\}} \\
& \leq\|\theta\|_{\infty}\left(1+\left\|D^{2} u\right\|\right) \chi_{\left\{\left\|D^{2} u\right\|<K\right\}} \\
& \leq\|\theta\|_{\infty}(1+K) \\
& \leq 2\|\theta\|_{\infty} K,
\end{aligned}
$$

where we use $K \geq 1$ in the last inequality. Subsequently, we derive

$$
\begin{aligned}
|g| & \leq|f|+\left\|D^{2} u\right\| \chi_{\left.\left\{\left\|D^{2} u\right\|<K\right\}\right\}}\left|H\left(D^{2} u, x, t\right)-\widetilde{H}\left(D^{2} u, x, t\right)\right| \\
& \leq|f|+2\left(\|\theta\|_{\infty}+1\right) K,
\end{aligned}
$$

which shows that $g \in L^{\gamma, q}\left(\Omega_{T}\right)$ since $\Omega_{T} \subset \mathbb{R}^{n+1}$ is a bounded cylinder domain and $f \in L^{\gamma, q}\left(\Omega_{T}\right)$. Moreover, we observe that

$$
\|g\|_{L^{\gamma, q}\left(\Omega_{T}\right)}^{q} \leq q \int_{0}^{\infty}\left(\mu^{\gamma}\left|\left\{(x, t) \in \Omega_{T}:|f(x, t)|>\frac{\mu}{2}\right\}\right|\right)^{\frac{q}{\gamma}} \frac{d \mu}{\mu}
$$




$$
\begin{aligned}
& +q \int_{0}^{\infty}\left(\mu^{\gamma}\left|\left\{(x, t) \in \Omega_{T}: 4\left\|D^{2} u\right\| \chi_{\left\{\left\|D^{2} u\right\|<K\right\}}>\frac{\mu}{2}\right\}\right|\right)^{\frac{q}{\gamma}} \frac{d \mu}{\mu} \\
& =2^{q} q \int_{0}^{\infty}\left(\mu^{\gamma}\left|\left\{(x, t) \in \Omega_{T}:|f(x, t)|>\mu\right\}\right|\right)^{\frac{q}{\gamma}} \frac{d \mu}{\mu} \\
& +2^{q} q \int_{0}^{\infty}\left(\mu^{\gamma}\left|\left\{(x, t) \in \Omega_{T}: 4\left\|D^{2} u\right\| \chi_{\left\{\left\|D^{2} u\right\|<K\right\}}>\mu\right\}\right|\right)^{\frac{q}{\gamma}} \frac{d \mu}{\mu} .
\end{aligned}
$$

Since

$$
\left|\left\{(x, t) \in \Omega_{T}: 4\left\|D^{2} u\right\| \chi_{\left\{\left\|D^{2} u\right\|<K\right\}}>\mu\right\}\right| \leq\left|\left\{(x, t) \in \Omega_{T}: 4 K>\mu\right\}\right|,
$$

we have

$$
\begin{aligned}
& \|g\|_{L^{\gamma, q}\left(\Omega_{T}\right)}^{q} \\
\leq & 2^{q}\|f\|_{L^{\gamma, q}\left(\Omega_{T}\right)}^{q}+2^{q} q \int_{0}^{\infty}\left(\mu^{\gamma}\left|\left\{(x, t) \in \Omega_{T}: 4 K>\mu\right\}\right|\right)^{\frac{q}{\gamma}} \frac{d \mu}{\mu} \\
\leq & 2^{q}\|f\|_{L^{\gamma, q}\left(\Omega_{T}\right)}^{q}+2^{q} q \int_{0}^{4 K}\left(\mu^{\gamma}\left|\left\{(x, t) \in \Omega_{T}: 4 K>\mu\right\}\right|\right)^{\frac{q}{\gamma}} \frac{d \mu}{\mu} \\
+ & 2^{q} q \int_{4 K}^{\infty}\left(\mu^{\gamma}\left|\left\{(x, t) \in \Omega_{T}: 4 K>\mu\right\}\right|\right)^{\frac{q}{\gamma}} \frac{d \mu}{\mu} \\
= & 2^{q}\|f\|_{L^{\gamma, q\left(\Omega_{T}\right)}}^{q}+2^{q} q \int_{0}^{4 K}\left(\mu^{\gamma}\left|\Omega_{T}\right|\right)^{\frac{q}{\gamma}} \frac{d \mu}{\mu}+0 \\
= & 2^{q}\|f\|_{L^{\gamma, q\left(\Omega_{T}\right)}}^{q}+2^{q} q\left|\Omega_{T}\right|^{\frac{q}{\gamma}} \int_{0}^{4 K} \mu^{q-1} d \mu \\
= & 2^{q}\|f\|_{L^{\gamma, q}\left(\Omega_{T}\right)}^{q}+2^{q}\left|\Omega_{T}\right|^{\frac{q}{\gamma}}(4 K)^{q},
\end{aligned}
$$

which implies

$$
\|g\|_{L^{\gamma, q}\left(\Omega_{T}\right)} \leq C\left(\|f\|_{L^{\gamma, q}\left(\Omega_{T}\right)}+1\right)
$$

with positive constant $C=C\left(n, \delta, \gamma, q, \theta,\left|\Omega_{T}\right|\right)$.

Considering

$$
H(M, x, t)=\frac{F(M, x, t)-G(M, x, t)}{\|M\|} \geq 0
$$

for all $M \in \mathcal{S}(n)$ and $(x, t) \in \Omega_{T}$, we have $\widetilde{H}(M, x, t) \geq 0$ for all $(x, t) \in \Omega_{T}$ due to (3.25). This shows that $\widetilde{H}\left(D^{2} u, x, t\right) \geq 0$ for all $(x, t) \in \Omega_{T}$. On the other hand, we know $G(M, x, t)$ and $\|M\|$ are convex in $M$, therefore $\widetilde{F}(M, x, t)=G(M, x, t)+\|M\| \widetilde{H}\left(D^{2} u, x, t\right)$ is also convex with respect to $M$.

Recalling Lemma 3.4 and using (3.34) and (3.36), we employ Theorem 3.3 with $G(M, x, t)$ replaced by $\widetilde{F}(M, x, t)$ and $f$ replaced by $g$, respectively, to obtain the desired estimate (2.5).

Finally, let us consider the Cauchy-Dirichlet problem with non-zero initial-boundary data as follows:

$$
\begin{cases}u_{t}+F\left(D^{2} u, x, t\right)=f(x, t), & \text { in } \Omega_{T}, \\ u=\varphi, & \text { on } \partial \Omega_{T},\end{cases}
$$

where the nonlinearity $F(M, x, t)$ is asymptotically regular with $G(M, x, t)$ satisfying the assumptions H1H3. Similar to Theorem 2.4, we can also establish the global Lorentz estimates of strong solutions for the initial-boundary problem (3.37). 
Theorem 3.5 For $\gamma>n+1$ and $0<q \leq \infty$, let $u \in W_{n+1}^{2,1}\left(\Omega_{T}\right)$ be any strong solution to the non-zero initial-boundary problem (3.37) with $f \in L^{\gamma, q}\left(\Omega_{T}\right), \varphi \in W^{2,1} L^{\gamma, q}\left(\Omega_{T}\right)$ and $\partial \Omega \in C^{1,1}$. Then there exists a small constant $\delta=\delta\left(n, \lambda, \Lambda, \gamma, q, \Omega_{T}\right)$ such that if $F\left(D^{2} u, x, t\right)$ is asymptotically regular with $G\left(D^{2} u, x, t\right)$ satisfying the assumptions $H 1-H 3$, we have $u_{t}, D^{2} u \in L^{\gamma, q}\left(\Omega_{T}\right)$ with the estimate

$$
\left\|u_{t}\right\|_{L^{\gamma, q}\left(\Omega_{T}\right)}+\left\|D^{2} u\right\|_{L^{\gamma, q}\left(\Omega_{T}\right)} \leq C\left(\|f\|_{L^{\gamma, q}\left(\Omega_{T}\right)}+\left\|\varphi_{t}\right\|_{L^{\gamma, q}\left(\Omega_{T}\right)}+\left\|D^{2} \varphi\right\|_{L^{\gamma, q}\left(\Omega_{T}\right)}+1\right)
$$

for some constant $C=C\left(n, \lambda, \Lambda, \gamma, q, \Omega_{T}\right)$. While $t=\infty$, the constants $\delta, C$ depend only on $n, \lambda, \Lambda, \gamma, \Omega_{T}$.

Proof. From the proof of Theorem 2.4, we know $u$ is a strong solution of

$$
u_{t}+\widetilde{F}\left(D^{2} u, x, t\right)=g(x, t), \quad \text { in } \Omega_{T},
$$

with data

$$
g=f-\left\|D^{2} u\right\| \chi_{\left\{\left\|D^{2} u\right\|<K\right\}}\left(H\left(D^{2} u, x, t\right)-\widetilde{H}\left(D^{2} u, x, t\right)\right) .
$$

Let $w=u-\varphi$, then $w$ is a strong solution of the zero initial-boundary problem

$$
\begin{cases}w_{t}+\widehat{F}\left(D^{2} w, x, t\right)=\hat{g}(x, t), & \text { in } \Omega_{T}, \\ w=0, & \text { on } \partial \Omega_{T},\end{cases}
$$

where

$$
\widehat{F}(M, x, t)=\widetilde{F}\left(M+D^{2} \varphi, x, t\right)-\widetilde{F}\left(D^{2} \varphi, x, t\right)
$$

and

$$
\hat{g}(x, t)=g(x, t)-\varphi_{t}-\widetilde{F}\left(D^{2} \varphi, x, t\right) .
$$

Obviously, $\widehat{F}(M, x, t)$ satisfies the assumptions H1-H3. Also, since $\widetilde{F}\left(D^{2} \varphi, x, t\right)$ is uniformly parabolic, we have

$$
|\hat{g}| \leq|g|+\left|\varphi_{t}\right|+\left|\widetilde{F}\left(D^{2} \varphi, x, t\right)\right| \leq|g|+\left|\varphi_{t}\right|+\widetilde{\Lambda}\left\|D^{2} \varphi\right\|,
$$

where $\widetilde{\Lambda}=\Lambda+\frac{\lambda}{2}$. Therefore, with the same argument as (3.36), we get

$$
\|\hat{g}\|_{L^{\gamma, q}\left(\Omega_{T}\right)} \leq C\left(\|g\|_{L^{\gamma, q}\left(\Omega_{T}\right)}+\left\|\varphi_{t}\right\|_{L^{\gamma, q}\left(\Omega_{T}\right)}+\left\|D^{2} \varphi\right\|_{L^{\gamma, q}\left(\Omega_{T}\right)}+1\right)
$$

with positive constant $C=C\left(n, \delta, \gamma, q, \theta,\left|\Omega_{T}\right|\right)$. Hence we apply Theorem 3.3 to $\hat{g} \in L^{\gamma, q}\left(\Omega_{T}\right)$ and $\widehat{F}(M, x, t)$ to discover $w_{t}, D^{2} w \in L^{\gamma, q}\left(\Omega_{T}\right)$ and

$$
\begin{aligned}
\left\|w_{t}\right\|_{L^{\gamma, q}\left(\Omega_{T}\right)}+\left\|D^{2} w\right\|_{L^{\gamma, q}\left(\Omega_{T}\right)} & \leq C\|\hat{g}\|_{L^{\gamma, q}\left(\Omega_{T}\right)} \\
& \leq C\left(\|g\|_{L^{\gamma, q}\left(\Omega_{T}\right)}+\left\|\varphi_{t}\right\|_{L^{\gamma, q}\left(\Omega_{T}\right)}+\left\|D^{2} \varphi\right\|_{L^{\gamma, q}\left(\Omega_{T}\right)}+1\right) .
\end{aligned}
$$

Then using $u=w+\varphi$ and (3.36), we arrive at the conclusion (3.38).

Acknowledgement The authors would like to thank the referees for their valuable comments. This paper was supported by NSFC grant 11371050, NSFC-ERC grant 11611530539 and the Fundamental Research Funds for the Central Universities of China grant 2016YJS154. The second author is also supported by ERCEA Advanced Grant 2014 669689-HADE, by the MINECO project MTM2014-53850-P, by Basque Government project IT-641-13 and also by the Basque Government through the BERC 2014-2017 program and by Spanish Ministry of Economy and Competitiveness MINECO: BCAM Severo Ochoa excellence accreditation SEV-2013-0323. 


\section{References}

[1] E. Acerbi, G. Mingione, Gradient estimates for a class of parabolic systems, Duke Math. J. 136 (2) (2007) 285-320.

[2] P. Baroni, Lorentz estimates for obstacle parabolic problems, Nonlinear Analysis 96 (2014) 167-188.

[3] S. S. Byun, Y. Cho, J. Oh, Global Calderón-Zygmund theory for nonlinear elliptic obstacle problems with asymptotically regular nonlinearities, Nonlinear Analysis 123-124 (2015) 150-157.

[4] S. S. Byun, M. Lee, D. K. Palagachev, Hessian estimates in weighted Lebesgue spaces for fully nonlinear elliptic equations, J. Differential Equations 260 (2016) 4550-4571.

[5] S. S. Byun, J. Oh, L. Wang, Global Calderón-Zygmund theory for asymptotically regular nonlinear elliptic and parabolic equations, Int. Math. Res. Not. IMRN 17(2015) 8289-8308.

[6] S. S. Byun, J. Oh, L. Wang, $W^{2, p}$ estimates for solutions to asymptotically elliptic equations in nondivergence form, J. Differential Equations 260 (2016) 7965-7981.

[7] L. A. Caffarelli, Interior a priori estimates for solutions of fully nonlinear equations, Ann. Math. 130 (2) (1989) 189-213.

[8] L. A. Caffarelli, X. Cabré, Fully Nonlinear Elliptic Equations, Vol. 43. American mathematical society colloquium publications, 1996.

[9] L. A. Caffarelli, M. G. Crandall, M. Kocan, A. Święch, On viscosity solutions of fully nonlinear equations with measurable ingredients, Comm. Pure Appl. Math. 49 (4) (1996) 365-397.

[10] L. A. Caffarelli, Q. Huang, Estimates in the generalized Campamato-John-Nirenberg spaces for fully nonlinear elliptic equations, Duke Math. J. 118 (1) (2003) 1-17.

[11] M. Chipot, L. C. Evans, Linearisation at infinity and Lipschitz estimates for certain problems in the calculus of variations, Proc. Roy. Soc. Edinburgh Sect. A. 102 (3-4) (1986) 291-303.

[12] M. G. Crandall, M. Kocan, A. Święch, $L^{p}$-theory for fully nonlinear uniformly parabolic equations, Comm. Partial Differential Equations 25 (11-12) (2000) 1997-2053.

[13] H. Dong, N. V. Krylov, X. Li, On fully nonlinear elliptic and parabolic equations with VMO coefficients in domains, St. Petersburg Math. J. 24 (1) (2013) 39-69.

[14] M. Foss, Global regularity for almost minimizers of nonconvex variational problems, Ann. Mat. Pura Appl. (4) 187 (2) (2008) 263-321.

[15] N. V. Krylov, On Bellman's equations with VMO coefficients, Methods and Applications of Analysis 17 (1) (2010) 105-122.

[16] N. V. Krylov, On the existence of $W^{2, p}$ solutions for fully nonlinear elliptic equations under relaxed convexity assumptions, Comm. Partial Differential Equations 38 (2013) 687-710.

[17] N. V. Krylov, M. V. Safonov, An estimate of the probability that a diffusion process hits a set of positive measure. Dokl. Akad. Nauk. SSSR 245, 235-255 (1979). English translation in Soviet Math Dokl. 20 (1979) 235-255 . 
[18] N. V. Krylov, M. V. Safonov, Certain properties of solutions of parabolic equations with measurable coefficients. Izvestia Akad Nauk. SSSR 40 (1980) 161-175.

[19] T. Kuusi, G. Mingione, New perturbation methods for nonlinear parabolic problems, J. Math. Pures Appl. (9) 98 (4) (2012) 390-427.

[20] C. Scheven, T. Schmidt, Asymptotically regular problems I: higher integrability, J. Differential Equations 248 (4) (2010) 745-791.

[21] C. Scheven, T. Schmidt, Asymptotically regular problems II: Partial Lipschitz continuity and a singular set of positive measure, Ann. Sc. Norm. Super. Pisa Cl. Sci. (5) 8 (3) (2009) 469-507.

[22] L. Wang, On the regularity theory of fully nonlinear parabolic equations, Bull. Amer. Math. Soc. (N.S.) 22 (1) (1990) 107-114.

[23] N. Winter, $W^{2, p}$ and $W^{1, p}$-estimates at the boundary for solutions of fully nonlinear, uniformly elliptic equations, Z. Anal. Anwend. 28 (2) (2009) 129-164.

[24] J. Zhang, S. Zheng, Lorentz estimate for nonlinear parabolic obstacle problems with asymptotically regular nonlinearities, Nonlinear Analysis 134 (2016) 189-203.

[25] J. Zhang, S. Zheng, Lorentz estimates for fully nonlinear parabolic and elliptic equations, Nonlinear Analysis 148 (2017) 106-125.

[26] W. P. Ziemer, Weakly differentiable functions. New York: Springer-Verlag(1989). 